\title{
A new missense mutation of fibrillin in a patient with Marfan syndrome
}

\author{
D R Hewett, J R Lynch, A Child, B C Sykes
}

\begin{abstract}
A patient with Marfan syndrome was shown to be heterozygous for a $G$ to $A$ transition at nucleotide 3952 of the FBNI gene. This would result in a cysteine to tyrosine substitution at amino acid 1223 in the fibrillin protein.
\end{abstract}

(F Med Genet 1994;31:338-339)

Marfan syndrome (MFS) is an autosomal dominant inherited disorder of connective tissue with a frequency of 1 in 10000 . It classically affects three systems, cardiovascular, ocular, and skeletal. ${ }^{1}$ The fibrillin gene FBNI is the disease causing locus for MFS. ${ }^{2-4}$ Fibrillin is a $350 \mathrm{kDa}$ protein that is an abundant component of 10-12 microfibrils. ${ }^{5} \mathrm{~A}$ full length cDNA copy of the FBNI mRNA was screened for mutations using 16 sets of overlapping primers and the polymerase chain reaction (PCR) to search for single stranded conformation polymorphisms (SSCP). ${ }^{6}{ }^{7}$ SSCP analysis on the Sau961 digestion fragments of the amplification product of primers G2S (5' AGGGAAGTATCAGTGTGCC 3') and G2AS (5' TCTTGCAGTCTGCATGCTG $3^{\prime}$ ) gave a band of aberrant mobility (data not shown). Sequencing of this region showed patient 2521 to be heterozygous for a $\mathrm{G}$ to $\mathrm{A}$ transition at nucleotide 3952. This would be predicted to change cysteine 1223 of the fibrillin polypeptide to a tyrosine. Screening of the unaffected members of the pedigree was performed using hybridisation of allele specific oligonucleotides to either the $G$ or the $A$ allele (fig 1).

None of the unaffected members of the pedigree was found to carry the A allele (fig 1). ASO screening of 100 controls and 58 other MFS patients showed it was neither a common cause of MFS nor a common polymorphic variant.

Patient 2521 is a 66 year old female whose parents were unaffected by MFS. She has bilateral dislocated lenses, myopia, and severe kyphoscoliosis. She has arachnodactyly in her left hand only, and one foot is longer than the other. She was diagnosed as having MFS when she gave birth to a daughter with a pronounced marfanoid habitus. The child had bilateral dislocated lenses, long fingers, hands, and feet, pectus excavatum, kyphoscoliosis, and poorly developed musculature. She died at
15 months from congenital heart disease complicated by bronchopneumonia. Necropsy showed that the pulmonary valve had only two cusps. There was no evidence of either mitral or aortic valve involvement in either patient 2521 or her affected daughter.

There was further evidence to suggest the mutation was causal. No sample was available from either of her unaffected parents, so it was not possible to show directly that the first appearance of the MFS phenotype in the pedigree coincided with a de novo mutation at cysteine 1223 . The pedigree was genotyped for the $(\mathrm{CA})_{\mathrm{n}}$ polymorphism MTS2 within intron 8 of the FBNI gene ${ }^{9}$ (fig 1). Each of the MTS2 alleles of patient 2521 was shared by at least one of her unaffected sibs. This showed that the mutation in patient 2521 was a de novo

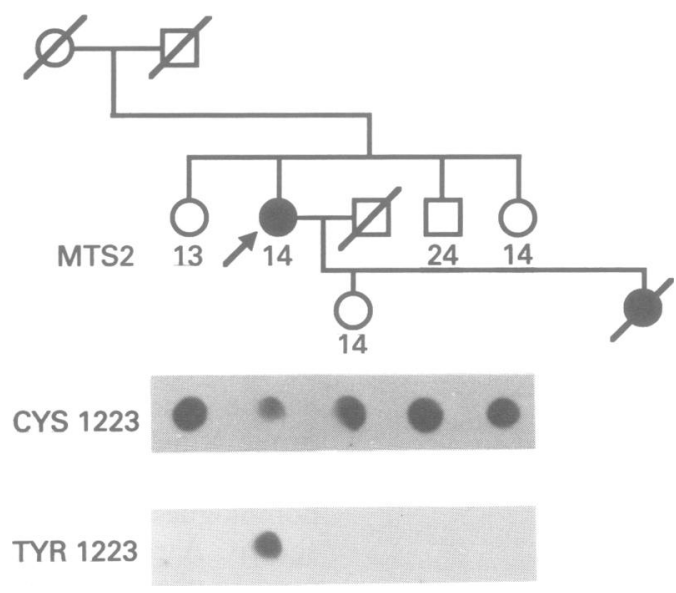

Figure 1 Screening of the proband (arrowed) and her pedigree by ASO hybridisation. G2S and G3S ( $5^{\prime}$ ATGATCTCTGGTCAGGCATT $3^{\prime}$ ) primed PCR amplification products of genomic DNA from pedigree members were filter immobilised. ${ }^{8}$ Amplifications were carried out according to standard conditions, ${ }^{6}$ with a temperature profile of: $94^{\circ} \mathrm{C}$ for 0.5 minutes; $58^{\circ} \mathrm{C}$ for 1.0 minute; $72^{\circ} \mathrm{C}$ for 1.0 minute (30 cycles). No samples were available from any of the dead persons. Directly below each extant family member is shown the autoradiograph from hybridisation of filter with oligonucleotides complementary to either the wild type (CYS 1223) allele or the mutant (TYR 1223) allele. The primers used were: CYS1223 ASO: (5'CCCGGCTGACAGCTACATT3'), and TYR1233 ASO: (5'AATGTAGCTATCAGCCGGG3'). The mismatched base of each oligonucleotide is
underlined. Washing was carried out at $58^{\circ} \mathrm{C}$ (TYR1223 ASO) or $60^{\circ} \mathrm{C}$ (CYS1223 ASO according to standard procedure. ${ }^{8}$ The MTS2 polymorphism genotypes are also shown on the pedigree. The MTS2 alleles were amplified and recognised under the conditions detailed in reference 9 . 
mutation that must have occurred in the germline of one of her parents.

The C1223Y mutation is located within the 15th EGF-like repeat of fibrillin. It is not the first cysteine substitution to be described in Marfan syndrome. At a recent meeting eight other MFS patients were shown to be heterozygous for missense mutations at key cysteine residues in fibrillin. ${ }^{10-15}$ All these cysteine substitutions were unique, and six of them occurred at highly conserved residues within EGF-like repeats. ${ }^{16}{ }^{17}$ There are 47 EGF-like repeats in the fibrillin molecule. ${ }^{231819}$ Fibrillin EGF-like repeat 15 is shown schematically in fig 2 , with the cysteines numbered from 1 to 6 ; cysteine 1223 occurs at cysteine number 5 in this repeat. The substitution of a tyrosine residue here would clearly disrupt the disulphide bond between cysteine number 5 and cysteine number 6 . The relative positions of the other six cysteine mutations that occur in other EGF-like repeats are also shown in fig 2.

The precedent for disease related substitutions at cysteine residues in EGF-like repeats is not limited to fibrillin and MFS. Similar mutations have been detected in human factor IX and low density lipoprotein receptor in

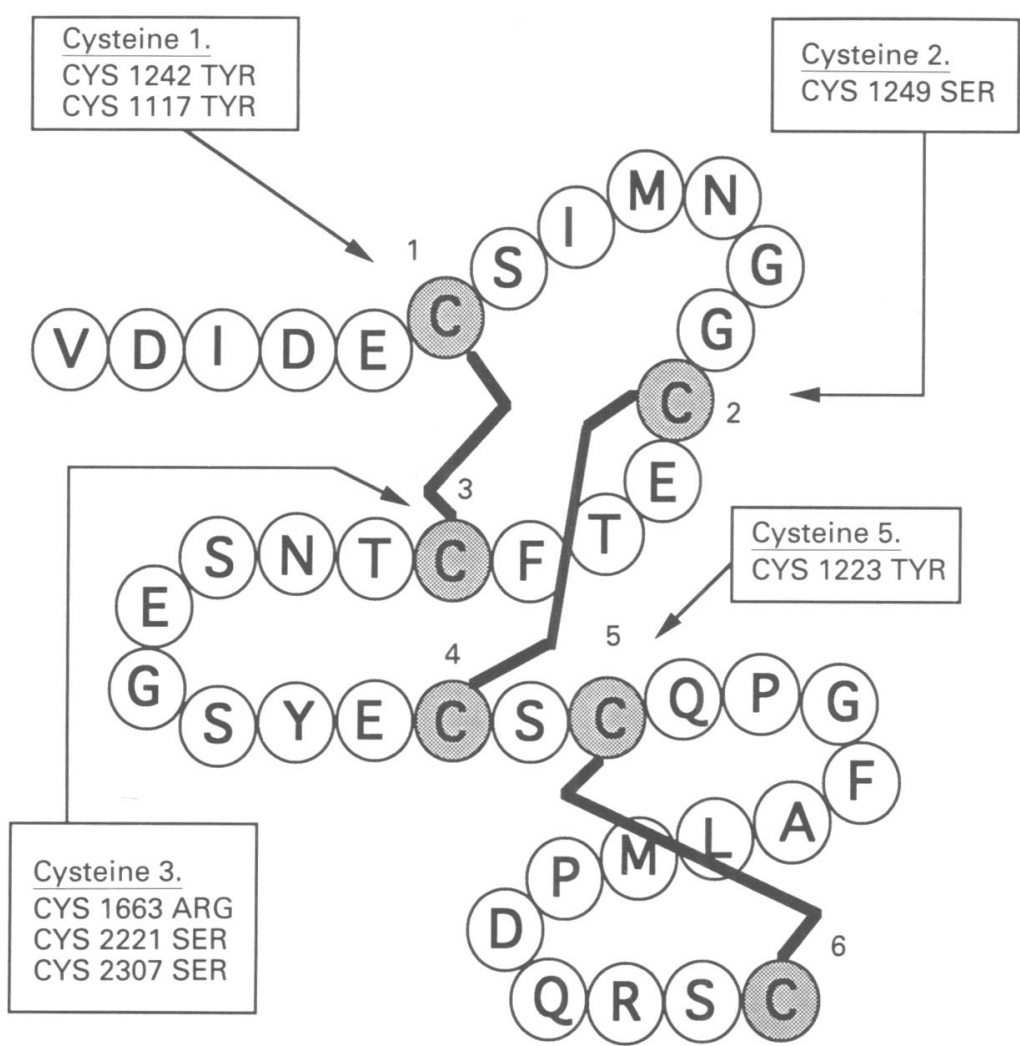

Figure 2 A schematic diagram of EGF-like domain number 15 of fibrillin. The six highly conserved cysteines are shaded. The cysteine residue involved in the mutation described in this patient (CYS 1223 TYR at cysteine 5) and others reported in different EGF-like domains are indicated by arrowed boxes. cases of haemophilia $\mathrm{B}^{20}$ and familial hypercholesterolaemia. ${ }^{21-23}$

We gratefully acknowledge access to sequence information, before publication, from Dr F Ramirez. This work was supported by a grant from the British Heart Foundation. D R Hewett is in receipt of a studentship from the Medical Research Council. Dr A Child gratefully acknowledges support from the Arthritis and Rheumatism Council and the GNS Trust. The Arthritis and Rheumatism Council and the GNS Trust. The
help of Dr Harold Bird, Professor Robert Dickson, and Dr help of Dr Harold Bird, Professor Robert Dickson, and Dr
Buckle, physicians caring for patient 2521, was much appreciated.

1 Pyeritz RE. The Marfan syndrome. In: Steinman B, ed. Connective tissue and its heritable disorders. New York: Connective tissue and its
Wiley-Liss, 1993;437-68.

2 Maslen CL, Corson GM, Maddox BK, Glanville RW, Sakai LY. Partial sequence of a candidate gene for the Marfan syndrome. Nature 1991;352:334-7.

3 Lee B, Godfrey M, Vitale E, et al. Linkage of Marfan syndrome and a phenotypically related disorder to two different fibrillin genes. Nature 1991;352:330-4.

4 Dietz HC, Cutting GR, Pyeritz RE, et al. Marfan syndrome caused by a recurrent de novo missense mutation in the fibrillin gene. Nature 1991;352:337-9.

5 Sakai LY, Keene DR, Engvall E. Fibrillin, a new 350-kD lycoprotein, is a component of extracellular microfibrils. † Cell Biol 1986;103:2499-509.

6 Saiki R, Scharf S, Faloona F, et al. Enzymatic amplification of $\beta$-globin genomic sequences and restriction site analysis for diagnosis of sickle cell anemia. Science lysis for diagn

7 Orita M, Suzuki Y, Sekiya T, Hayashi K. Rapid and sensitive detection of point mutations and DNA polymorphisms using the polymerase chain reaction. Genomics 1989;5:874-9.

8 Kazazian HH. Diagnosis of monogenic disease. In: Ehrlich HA, eds. PCR technology. London: Stockton Press, 1989:165-9.

9 Pereira L, Levran O, Lynch JR, Sykes B, Ramirez F. Typing of the FBNI locus (FBNI) using five VNTR polymorphisms. Genomics (submitted)

10 Dietz HC, Saraiva JM, Pyeritz RE, Cutting GR, Francomano CA. Clustering of fibrillin (FBNI) missense mutations in the Marfan syndrome patients at cysteine residues in EGF-like domains. Hum Mutat 1992;1:366-75.

11 Pearson M, Tynan K, Aoyama T, Gasner C, Furtmayr H, Francke U. Mutation in TGF- $\beta 1$-like domain of FBNI associated with a MFS/CCA phenotype. Am $\mathcal{F}$ Med Genet 1993;47:143-60.

12 Tynan K, Comeau K, Pearson M, et al. Search for molecular defects in FBNI in Marfan syndrome. Am $\mathcal{F}$ Med Genet 1993;47:143-60.

13 Rantamaki T, Lohnqvist L, Kainulainen K, Peltonen L. Amplifiable markers in the diagnostics of Marfan syndrome. Am $\mathcal{F}$ Med Genet 1993;47:143-60.

14 Dietz HC, Pyeritz RE, Puffenberger EG, et al. Marfan phenotype variability in a family segregating a missense mutation in the epidermal growth factor-like motif of the fibrillin gene. $\mathcal{f}$ Clin Invest 1992;89:1674-80.

15 Sykes B. Marfan gene dissected. Nature Genet 1993;3:99 100.

16 Baron M, Norman DG, Campbell ID. Protein modules. Trends Biochem Sci 1991;16:13-17.

17 Stenflo J. Structure-function relationships of epidermal growth factor modules in vitamin $\mathrm{K}$-dependent clotting factors. Blood 1991;78:1637-51.

18 Pereira L, D'Alessio M, Ramirez F, et al. Genomic organization of the sequence coding for fibrillin, the defective gene product in Marfan syndrome. Hum Mol Genet gene product

19 Corson GM, Chalberg SC, Dietz HC, Charbonneau NL Sakai LY. Fibrillin binds calcium and is coded by cDNAs that reveal a multidomain structure and alternatively that reveal a multidomain structure and alternative

20 Gianelli PM, High KA, Sommer S, et al. Haemophilia B database of point mutations and short additions and deletions - third edition. Nucleic Acids Res 1992, 20(suppl):2027-63.

21 Hobbs HH, Russell DW, Brown MS, Goldstein JL. The LDL receptor locus in familial hypercholesterolemia. Annu Rev Genet 1990;24:133-70.

22 Brown MS, Goldstein JL. A receptor mediated pathway for cholesterol homeostasis. Science 1986;232:34-47.

23 Hobbs HH, Brown MS, Goldstein JL. Molecular genetics of the LDL receptor gene in familial hypercholesterolemia. Hum Mut 1992;1:445-66. 